\title{
IMPROVED AUTHENTICATION USING ARDUINO BASED VOICE AND IRIS RECOGNITION TECHNOLOGY
}

\author{
M. Usha Rani ${ }^{1}$, J. Goutham², M. Parthiban ${ }^{3}$ \\ ${ }^{I}$ M.E. Network Engg, Velammal College of Engineering and Technology, Tamil Nadu, India \\ ${ }^{2}$ B.Tech, Information Technology, Velammal College of Engineering and Technology, Tamil Nadu, India \\ ${ }^{3}$ MBBS, PES Institute of Medical Science and Research Institute, Andhra Pradesh, India
}

\begin{abstract}
Today authentication is the important one to improve the security in various applications Here the proposed system uses three steps to allow the process that is initially enter the password and then give the voice as the input using the arduino microcontroller it verifies the voice and then iris identification of the user. The user has to follow these steps and then only permit to continue the process. Based on the proposed system it highly protects your site from the hackers improve the efficiency also. The Iris recognition utilizes iris biometric technology to update the human's iris image, verify it because this is the most secured method to improve the resources and the data become so confidential. Particularly this proposed system used to improve the Bank transaction via internet communication using the low cost arduino microcontroller. This system improves the authentication using the three step method by input as a text password, voice input, Iris recognition technology
\end{abstract}

Keywords: Arduino Microcontroller, Password, Voice recognition, Iris recognition

\section{INTRODUCTION}

The Arduino is an open-hardware platform ready to be used and to create prototype based on easy to use and flexible software and hardware.[6] Arduino can get information from the environment through its pines from a lot of sensors and it can control lights, motors and other devices. This type of iris recognition biometric technology is the strong and scientific demand for the proliferation of systems for iris identification and recognition. This is the best because the face and, finger print and other biometric trails they need so many mathematical calculation and computer vision process on the research area. Voice recognition is the technology by which sounds, words or phrases spoken by the humans are converted into the electrical signals, and these signals are transformed into coding to which the control to be assigned will work on it.

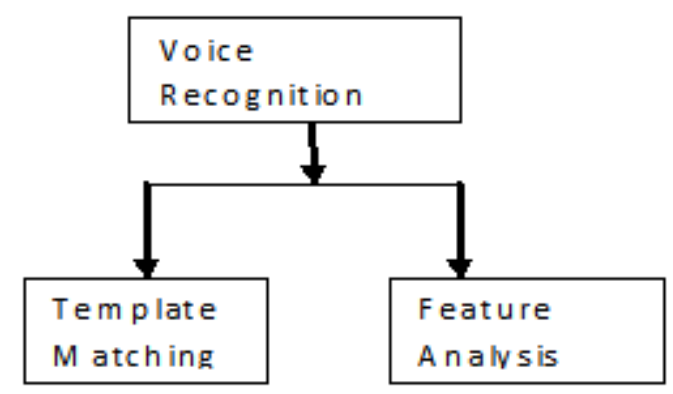

Fig 1 Voice Recognition types

Based on the approaches the template matching is the technique has the high accuracy when it used properly. Here to introduce the same template matching process.

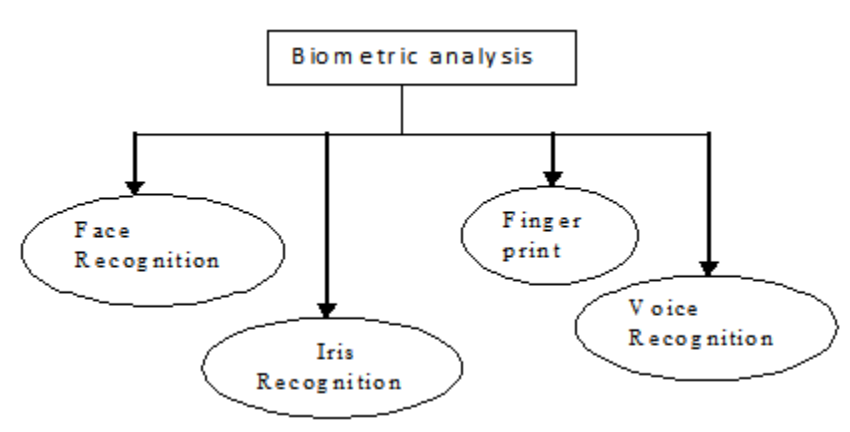

Fig 2 Biometric technologies

Another area biometric iris recognition authentication technique which is very useful with is physical security to the applications which need more security to allow the secured users only allow the process. This helps to alleviate problems associated with swipe card access where some systems have to be manually programmed with specific card numbers and robust processes need to be in place to ensure access lists are regularly reviewed. Swipe cards are also easily lost, stolen or borrowed.

\section{LITERATURE REVIEW}

There is a use of iris recognition technology is within the computing environment. Today the security is a very important one to protect the data from the ethical aspects and from the hackers. For example the bank transactions are have more and more secure detail information in that case the bank need more security in order protect their data information and other details. [3] The user and the bank working staff's being able to access the network with their 
username and password is the only authentication steps in all application processes. In this approach we propose three step security method incase to improve the security level with low cost implementation. Why this three step verification is important means if the username and password may be stolen by any one means it affects the major problem in their transaction.

[2] By providing the Voice recognition technology and the iris recognition technology with addition to the username and password method. Using this techniques the voice and the iris are the more secured method and it is impossible to replicate the same voice input password and the same iris as a password and plus there is no more option to set the same voice and iris in that time of password needed on the network and these security steps are impossible to do by the hackers. For these advance method the transactions are become so safe and so secure at the time of transfer information through the network. [6] In this the Arduino microcontroller is make a important role to with the integration of EasyVR Shield and the Iris Recognition shield. Initially the faculty members of the organization or if network transactions means the user of the company and any other secured applications.

\section{PROPOSED SYSTEM}

The proposed system explains the three step factor to improve the authentication scheme that is password entering, Voice recognition, Iris Recognition. These are the requirements to improve the authentication in the system. This block describes the overall proposed algorithm of the three- factor authentication system.

This very secured and important method to improve the system by implementing this with low cost Arduino Microcontroller. Initially we tested with voice input to control the LED it is working and then we extend that work provide as a password to the system. For this initially the is voice is stored in the database and every time the user give the voice input it checks with the database voice input if it corrects then proceed to allow the user to access the system or the job in the network.

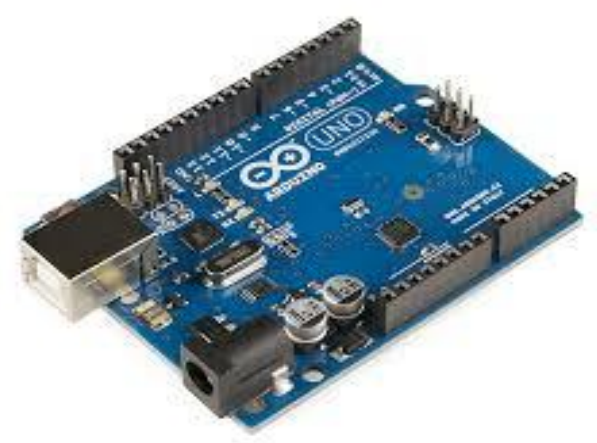

Fig 3: Arduino Micro Controller

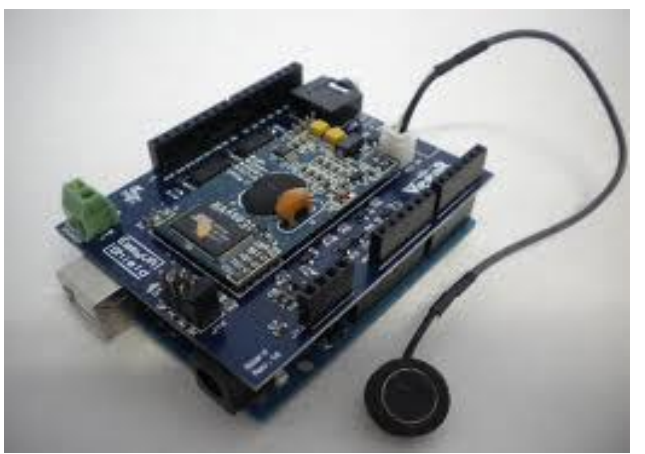

Fig 4: Arduino Microcontroller with EasyVR Shield

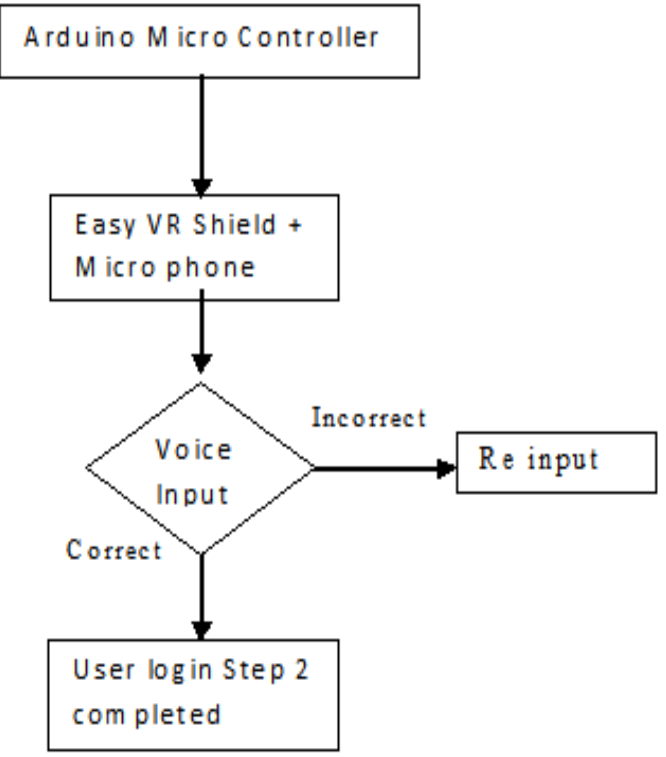

Fig 5 Flow diagram of Voice Recognition

The voice recognition is used to improve the security that here the user has to give the input via headphone and the input is forwarded to the Easy VR Shield using the arduino microcontroller. [2] Initially the default user voice input password is define and stored in the database .whenever there is a need to access the process then first enter the password and then give the user input then if correct means then only it forward to the next step of Iris Recognition process

Iris recognition is the process of recognizing a person by analyzing the apparent pattern of her or his iris.

- In windows: Windows $7 \& 8$

- For Android: Android OS 3.1

These two platforms are supported by this technology. So this is platform independent and this also makes the one more advantage to use this three step authentication for the secured transaction in the network.

The iris identification module is responsible for recognizing the iris of the user. During the identification stage, the biometric sensor in the iris recognition module captures the 
characteristics of the user and it to be identified and then converts it into the system format and then assigned as the template. [3] which compares it against the stored template in the database to determine whether the two templates match then only it will continue to the next step otherwise there is an error will be displayed.

The identification can be in the form of verification, authenticating a claimed identity or recognition, determining the identity of a person from a database to identify the person is correct or not. In a verification system, the captured iris is stored in the database and saved as a template to provide identity to the user and the faculty members in the bank or any company while enter into the network or in the system. Every time the user there is in a need to show the iris in front of the iris recognition module which is connected with the arduino microcontroller board. At the time the captured iris is verified with the template iris if it is matched then only the user is allowed to do their process in the network.

Iris Recognition technology methods:

Processing of capture the Iris into 3 steps

i. Capture the iris image

ii. Optimizing the iris image and assign as a template image

iii. Store the image in database to compare when user gives an input

These are all the three steps that is used to capture the image from the camera and then process it to the module to get the input from the user module.

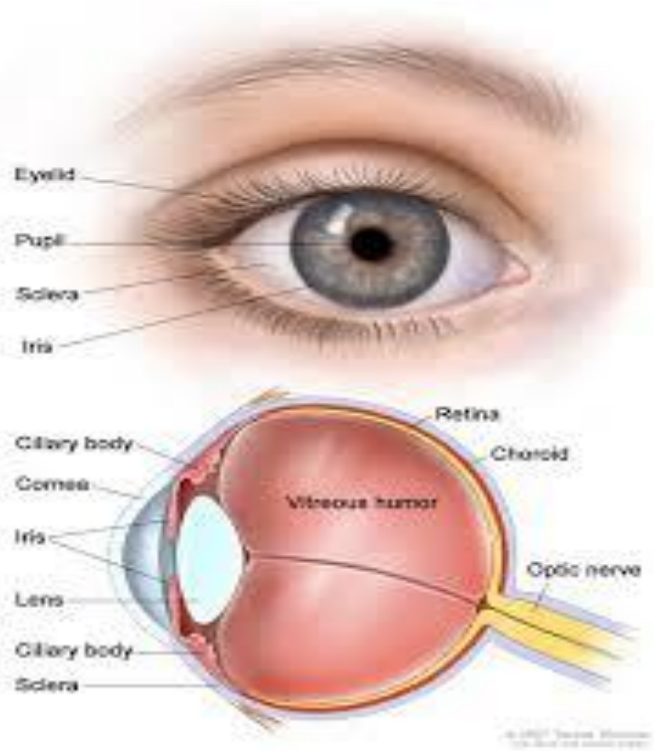

Fig 6: Eye diagram to display the iris part

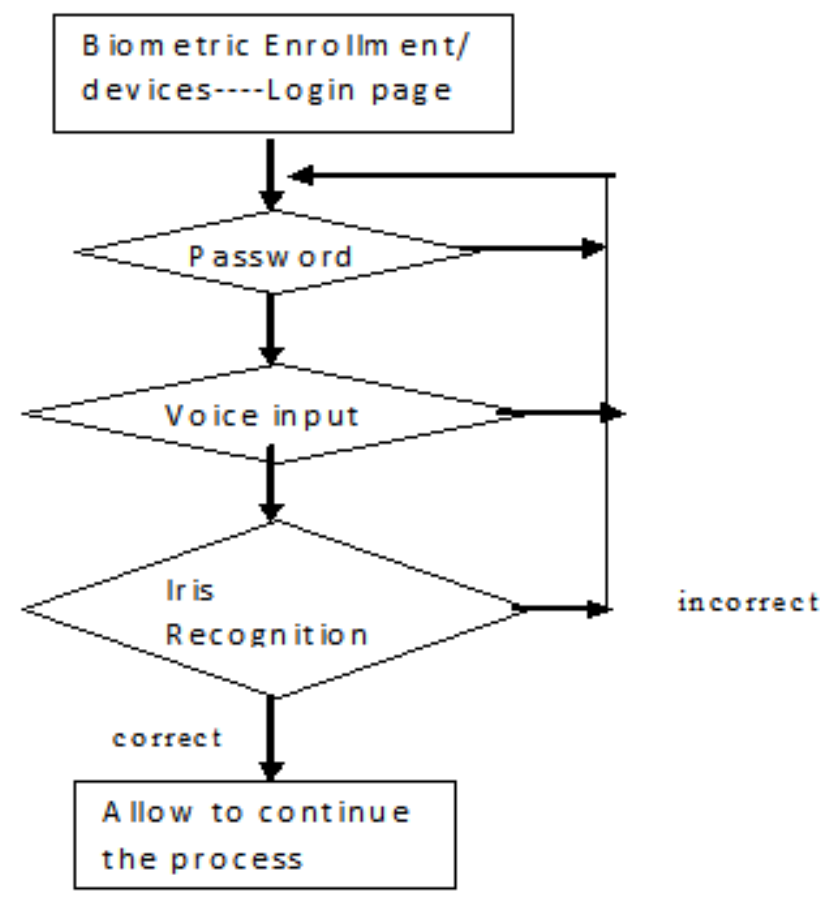

Fig 7 Overall process flow diagram

This is the flow diagram of the part of Iris recognition technique part. Initially the iris to be captured form the user and it to be stored in the database as the template . Whenever there is a need to access the network or the system the iris is captured form the user the captured iris and the template iris is matched then only the system allowed continuing the process. Before that the voice and the password are to be correct then only capture the iris and the process continue on.

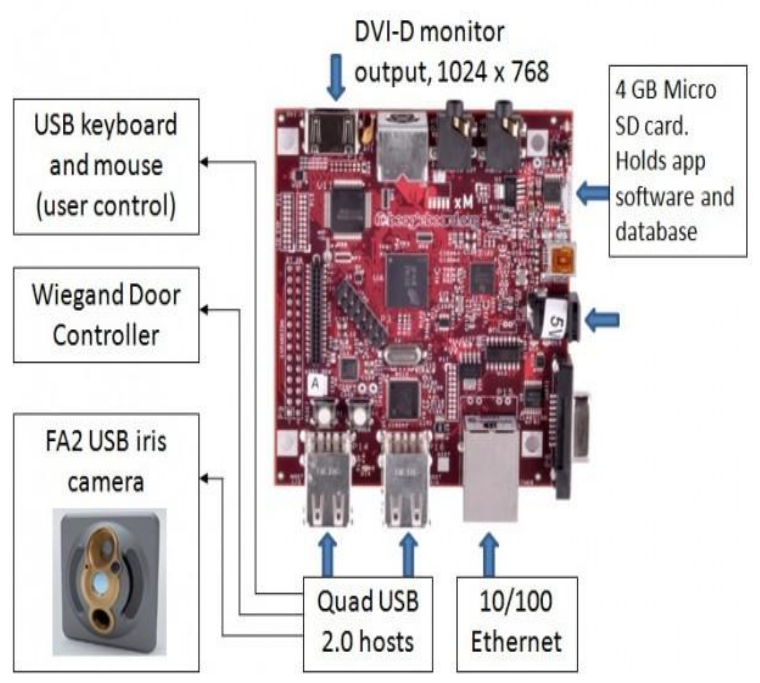

Fig 8: Iris Recognition module

The Iris recognition module contains the combination of DVI- D Monitor, 4GB Micro SD holds app software and the database, USB Keyboard and mouse for the user control, FA2 USB Iris Camera, Quad USB 2.0 hosts, 10/ 100 Ethernet port also available in the iris recognition module. 
The uniqueness of the iris and low probability of a false acceptance or false rejection all contribute to the benefits of using iris recognition technology. [3] It provides an accurate and secure method of authenticating users onto company systems, and has the speed required minimizing user frustration when accessing the secured systems.

\section{CONCLUSIONS}

Finally the improved authentication is provided by the three proposed methods Using the Arduino Microcontroller, EasyVR shield and with the Iris recognition shield. By using the Iris means don't worry about the password hackers this is the strong and effective password to the user. These are increasing the security to the system by online and offline network. Provides better security to the bank transactions and the Military applications, in need of secure information transaction. The already existing system for example by only using the password and the PINs are very risk if it may be known to the hackers the total information will be taken by the hackers. So in today the security is the important one to the transaction. Here the three-factor authentication system is implemented with the low cost implementation.

\section{FUTURE ENHANCEMENTS}

In future have to extend the proposed system to develop that process based on the mobile application environment for that increase the security as efficiently to everyone for secure communication in the mobile phones using the mobile application in the android and windows OS.

\section{REFERENCES}

[1]. M. Baloul, E. Cherrier, and C. Rosenberger. Challengebased speaker recognition for mobile authentication. In International Conference of the Biometrics Special Interest Group (BIOSIG), pages 1\{7, 2012

[2]. Daugman John “ How Iris Rcognition Works" IEEE Transaction on Circuits and systems for Video Technology 14 (1) (January 2004) : 21-30

[3]. NTSC Subcommite on Biometrices " Biometrics Overview" Web( August:006

[4]. M.M.Gifford, D.J. McCartney and C.H.Seal, "Networked biometrics systems: requirements based on iris recognition”, BT Technol. Journal, Vol. 17, n², April 1999. [5]. R.P.Wildes, J.C. Asmuth, G.L. Green and S.C. Hsu, A system for automated iris recoginition", IEEE paper 1994 [6]. www. arduino.cc.

\section{BIOGRAPHIES}

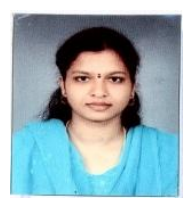

Usha Rani. M, M.E Network Engineering, Velammal College of Engineering and Technology, Madurai

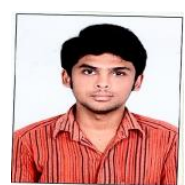

Goutham. J , B.Tech Information Technology ,Velammal College of Engineering and Technology, Madurai
Parthiban. M, MBBS, PES Institute of Medical Science and Research Institute, Andhra Pradesh 